Nieren-Epithelien vorkommt, müssen wir, so nahe die Versuchung einer solchen Annahme liegt, von weiteren Untersuchungen entscheidende Aufschlüsse erwarten.

\title{
Erklärung der Abbildungen auf Taf. I.
}

Fig. 1. Mausniere. Supravitale Färbung der näehst dem Innensaum gelegenen Granula durch Neutralroth.

Fig. 2. Mausniere. Dasselbe; stellenweise Ausdehnung der GranulaFärbung nach aussen.

Fig. 3. Mausniere. Supravitale Färbung der Granula durch Neutralroth. Kerne nach allen Seiten von gefärbten Granula umgeben.

Fig. 4. Menschliche Niere. Supravitale Färbung der Granula am Innensaum.

Fig. 5. Mausniere. Vitale Injection einer gesättigten Lösung von Methylenblau in das Unterhautzellgewebe. Färbung der Granula am Innensaum.

Fig. 6. Dasselbe. Ausdehnung der Granula-Färbung nach Aussen.

Fig. 7. Dasselbe. Lichtblaue Färbung der Stäbchen, in welche intensiv gefärbte Granula eingebettet liegen.

Fig. 8. u. 9. Dasselbe. Isolirte Zellen.

Fig. 10. Menschliche Niere. Supravitale Färbung der Granula am Innensaum.

Fig. 11. Mausniere. Injection von indigschwefelsaurem Natron in das Unterhautzellgewebe. Granula-Färbung am Innensaum.

Fig. 12. Mausniere. Injectiön von gesättigter Lithioncarmin-Lösung. Gefärbte Granula an Bürsten- und Innensaum.

Fig. 13. Dasselbe. Durch rothe Granula eingesäumte helle Felder.

Fig. 14. Menschliche Niere. Formol-Chromsäure-Färbung mit DreifarbenGemisch von Pianese. Differenzirung mit schwachsaurem Alkohol. Plasmosomen hellroth gefärbt; zwischen ihnen dunkelgefärbte Granula.

\section{II. \\ Ueber die Emigrations-Fähigkeit der Lymphocyten.}

(Aus dem Laboratorium des städtischen Gesundheitsamts in Stockholm.) Von

Dr. Johann Almkvist in Stockholm. (Hierzu Taf. II.)

Seitdem Cohwheim 1867 die Emigrations-Fähigkeit weisser Blutkörperchen experimentell zeigte, ist das Verhältniss emiArchiv f. pathol. Anat. Bd. 169. Hft. 1. 
s
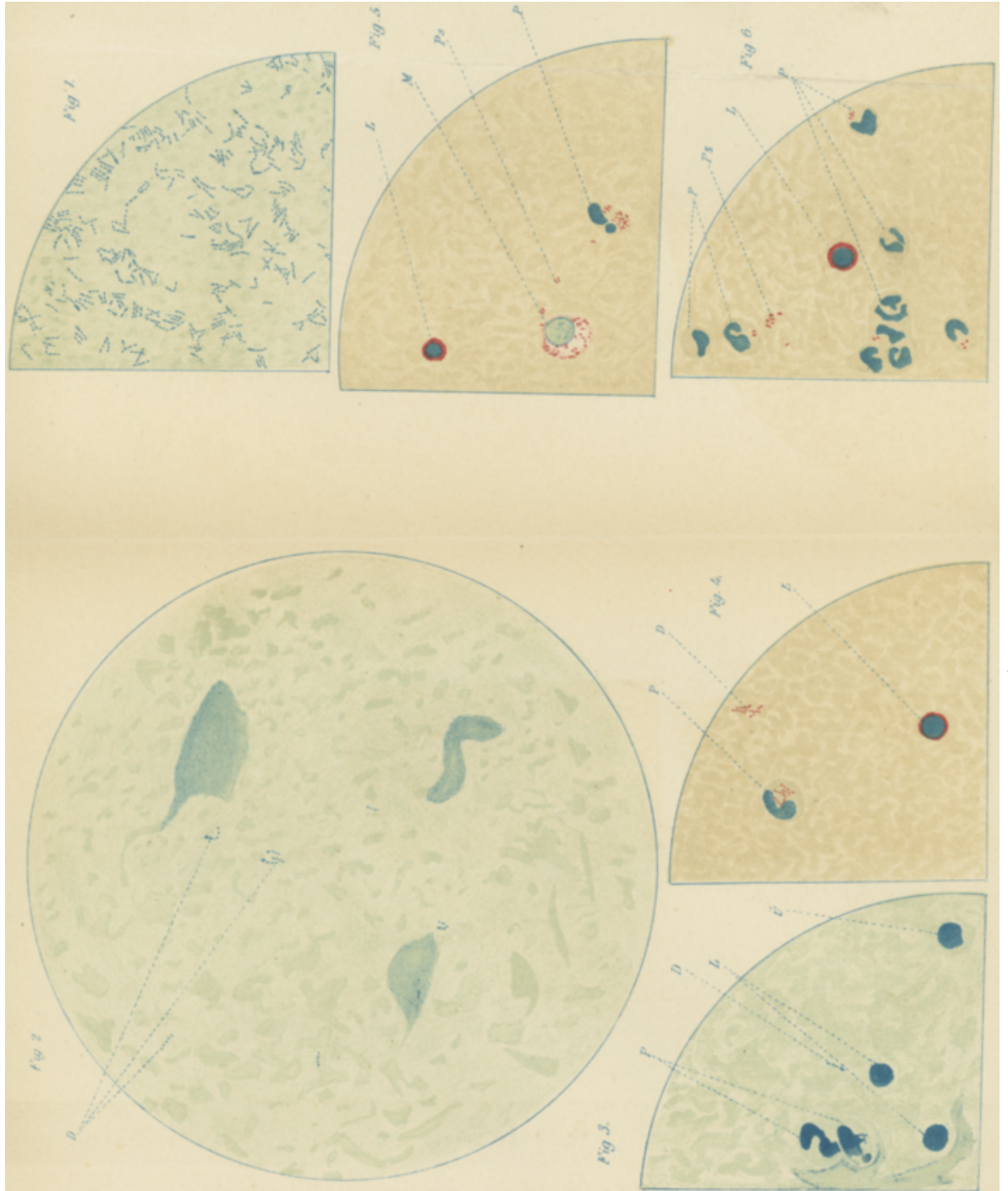
grirter Blatzellen und fixer Gewebszellen bei der Entzündung fleissig studirt und lebhaft discutirt worden. Cohnheim und seine Schule sind so weit gegangen, den fxen Zellen bei dem Entzündungs-Processe eine völlig passive, nur regenerative Rolle zuzuschreiben und betrachten alle in Entzündungs-Heerden auftretenden kleinen Rundzellen als aus den Gefässen des betreffcnden Theils extravasirte farblose Blutkörperchen.

Gegen diese einseitige hämatogene Lehre hat sich bald eine Reaction geltend gemacht. Als unumstössliches Resultat aus diesen späteren Untersuchungen ist hervorgegangen, dass auch die fixen Gewebszellen eine active Betheiligung an dem Entzündungs-Process nehmen. So ist die Virchow'sche Lehre theilweise rehabilitirt worden.

In einem Punkte ist aber doch Cohnheim's Ansicht bestätigt worden, nehmlich dass die typischen "Eiterkörperchen" nicht durch Proliferation fixer Gewebszellen gebildet werden, sondern durch Emigration farbloser Blutkörperchen, und zwar der sogenannten polynucleären Leukocyten.

Von den neueren Untersuchungen sind besonders diejenigen Marchands $8,9,10,11,12,13$ und seiner Nachfolger Ha mmerl ${ }^{6}$ v. Büngner ${ }^{3}$ und Borst ${ }^{2}$ von grossem Interesse.

Diese Unsersuchungen, die theils bei Einheilungs-Processen, theils auf dem gereizten Netz von Meerschweinchen gemacht sind, haben übereinstimmend gezeigt, dass dei der Entzündung, neben der Emigration der multinucleären Leukocyten, theils grössere, theils kleinere runde Zellen aus dem Bindegewebe entstehen. Die grösseren sind den grossen, - uninucleären Leukocyten, die kleineren den kleinen Lymphocyten vollkommen ähnlich. Weiter hat sich auch gezeigt, dass die Emigration früher, die Proliferation später eintritt. Die grossen Zellen haben eine sehr ausgesprochene Phagocytose und werden auch grosse Phagocyten genannt. Die kleinen "lymphocytoiden" Zellen scheinen in die Gefässe hineinwandern zu können und wirkliche Lymphocyten zu werden.

Durch diese Untersuchungen ist aber nur die Möglichkeit der Entstehung kleiner, runder, Lymphocyten ähnlicher Zellen aus Bindegewebszellen bewiesen. Wie oft dies geschieht, weiss man nicht - und ebensowenig, ob alle oder nur einige Bindegewebszellen einer solchen Proliferation fähig sind. 
Auch schreibt Baumgarten ${ }^{1}$, der doch den fixen Zellen in der Entzündung eine bedeutende Rolle zuschreibt -, dass er, in seinen "sehr zahlreichen, die Herkunft der in Entzündungs. Heerden auftretenden zelligen Elemente betreffenden Untersuchungen aus wuchernden Bindegewebs- und Endothelzellen nur zellige Elemente vom Habitus der sogen. Epithelioidzellen hervorgehen gesehen hat."

Uebrigens macht F. Marchand selbst in seinem neulich erschienen Buch „Der Process der Wundheilung ${ }^{413}$ eine gewisse Einschränkung in der Gemeingültigkeit seiner Entdeckung. Auf S. 132 fasst er das Resultat der Untersuchungen über diese "leukocytoiden" Zellen zusammen, indem er die Annahme berechtigt findet, dass die Blutgefässe regelmässig von einer Anzahl Zellen begleitet werden, die die Fähigkeit besitzen, Elemente von der Beschaffenheit der Lymphocyten und der grossen einkernigen Leukncyten zu produciren, dass ferner die Möglichkeit vorliegt, „dass diese Zellen in die Blutbahn gelangen, und auf diese Weise wirkliche Leukocyten bilden. Die Umwandlung in gewöhnliche multinucleäre Zellen, die Bildung der Granula in den Zellen, die Veränderungen der Kernformen sind secundäre Vorgänge, welche zum Theil in der Blutbahn, zum Theil wohl in bestimmten Organen, z. B. dem Knocheninark, stattfinden." „Durch diese Auffassung wird allerdings die Lehre von der Specificität der Leukocyten modificirt, immerhin bleibt der Formenkreis dieser Elemente im Wesentlichen derselbe“ „Die Herkunft der Exsudatzellen, d. h. der bei der acuten Entzündung im Exsudat auftretenden multinucleären Zellen, durch Auswanderung aus den Gefässen, wird durch diese Auffassung in keiner Weise beeinträchtigt.“

„Eine Umwandlung leukocytoider Zellen in Bindegewebszellen halte ich nicht für wahrscheinlich. Auch erscheint mir eine Verallgemeinerung der Herleitung der Lenkocytoiden von den Bindegewebszellen überhaupt nicht zulässig."

„Wenn man weiss, mit wie grossen Schwierigkeiten der sichere Nachweis der Herkunft so verschiedenartiger Zellformen, wie die der Leukocyten, verbunden ist, wird man es verstehen, wenn ich die vorgetragene Auffassung nur mit einem gewissen Vorbehalt ausspreche." 
Marohand selbst, der doch die betreffenden Untersuchungen am besten kennen muss, glaubt also, dass es eigentlich nur gewisse Zellen der Gefässwände sind, die der Proliferation leukocytoider Zellen fähig sind.

Dass weiter lymphocyten im Gewebe auch auf andere Weise, d. h. durch Braigration aus den Gefässen entstehen können, ist natürlich durch diese Untersuchungen auf keine Weise ausgeschlossen. Und gegen eine solche Entstehung von Lymphocyten im Gewebe durch Emigration aus den Gefässen giebt es auch - so viel ich sehen kann - keine wirklichen Beweise.

Denn dass der bekannte Versuch Neumann's einen Beweis gegen die Emigrations-Fähigkeit der Lymphocyten ist, kann ich nicht finden. Es gelang Neumann nicht, bei einem Patienten mit lymphatischer Leukämie, dessen Blatbahn fast aussohliesslich kleine Lymphocyten führte, mittelst eines Vesicans Lymphocyten-Eiter in der enstehenden Vesicatorblase za erzeugen. Dies beweist doch nichts Anderes, als dass das ron Neumann angewandte Vesicans keine chemotoktische Wirkng auf die Lymphocyten ausübte.

In seiner Arbeit über einkernige Zellen im gonorrhoischen Secret ${ }^{15}$ spricht Pappenheim nicht ohne weiteres den Lymphocyten eine Auswanderungs-Fähigkeit ab, sondern nimmt die Frage in Erwägung. In einer späteren Arbeit ${ }^{16}$ ) dagegen stellt er die Emigrations-Unfähigkeit der Lymphocyten als "Dogma" auf. Er sagt (S. 375 dieses Archivs, Bd. 165): "Es ist einer der wichtigsten Leitsätze der modernen Hämatologie, dass den ungekörnten basophilen Lymphocyten in functionelier Hinsicht chemotoktische Eigenschaften and also auch active Emigrations-Fähigkeit abzuerkennen sind."

Da ich indessen während meiner experimentellen Arbeiten, die eigentlich zu anderen Zwecken gemacht wurden, Gründe für die Emigrations-Fähigkeit der Lymphocyten gefunden zu haben glaube, erlaube ich mir diese Experimente hier kurz mitzutheilen.

Seit August 1901 bin ich mit vergleichenden Studien über den Diphtherie-Bacillus und Pseudodiphtherie-Bacillus beschäftigt. Besonders habe ich dabei das Verhältnis dieser beiden Bacillen zu der Immunitäts-Reaction. Pfeiffer's untersucht. 
Erstens habe ich darum bei 2 Kaninchen subcutane Injectionen von getödteten Diphtherie- und PseudodiphtherieCulturen während langer Zeit in immer kürzer werdenden Intervallen gemacht. Dann wurde in verschiedenen sterilen Eprouvetten Blutserum von diesen beiden Kaninchen und daneben von einem normalen Kaninchen genommen. Von solchem Serum wurde nachher $1 / 10 \mathrm{~cm}^{3}$ in bestimmter Proportion mit lebenden Diphtherie-Culturen sehr genau gemischt und in die Peritonaealhöhle eines Meerschweinchens miteiner sterilen Capillarpipette injicirt. Ein anderes Mal wurde eine gleiche Quantität desselben Serums in gleicher Weise mit PseudodiphtherieBacillen gemischt und injicirt. Auch die anderen beiden Blutsera wurden in gleicher Weise behandelt.

Nach der Injektion wurde während 3 Stunden jede 10. bis 20. Minute mit einem sterilen capillären Glasrohr, welches durch die Bauchwand in die Peritonealhöhle hineingesteckt wurde, eine Probe des darin hervorgerufenen Exsudates herausgenommen.

Dies geschieht ja eigentlich, um die Veränderung der Bacillen während dieser Zeit zu beobachten, aber dass man gleich zeitig das durch Reizung des Peritonaeums hervorgerufene inflammatorische Exsudat studiren kann, ist ja ohne weiteres klar.

Wenn man beim Herausnehmen einer solchen Probe aus der Peritonaealhöhle unvorsichtig oder mit einem unzweckmässigen Rohr durch die Bauchwand sticht, kann man leicht eine Blutung in die Peritonaealhöhle hinein erregen. Wenn diese Blutung grösser war, habe ich immer dieses Meerschweinchen verworfen und eine neue Injections-Serie gemacht. War weiter eine auch noch so minimale Blutung entstanden, so dass man bei mikroskopischer Untersuchung der herausgenommenen Probe mehrere oder vereinzelte rothe Blutkörperchen sah, so wurden diese Proben beim Studium des hervorgerufenen Exsudates unbeachtet gelassen.

Auch wurde die Injektions-Mischung vor dem Einspritzen untersucht und immer wurde festgestellt, dass diese Mischung von Serum und Bakterien-Cultur keine Blutkörperchen enthielt.

Das Studium des Exsudates fand also nur an solchen Proben statt, wo man eine Beimischung von Blut vollkommen sicher ausschliessen konnte. 
Von den verschiedenen Proben, die man aus der Peritonaealhöhle herausnimmt, bestehen die ersten makroskopisch nur aus einer vollkommen klaren, ungefärbten Flüssigkeit. Nach ungefähr einer Stunde oder etwas früher, manchmal auch etwas später, beginnt die herausgenommene Flüssigkeit mehr und mehr trüb auszusehen, und die letzten Proben sind bedeutend trüber geworden oder sehen manchmal aus wie dünner Eiter.

Die herausgenommenen Proben wurden auch mikroskopisch untersucht. Darum wurde die Flüssigkeit auf verschiedenen, gut gereinigten Objectträgern ausgestrichen, in der $W$ ärme fixirt und mit verschiedenen Farben (Löffler's Methylinblau, Papponheim's Methylgrün-Pyronin-Methode, Ehrlich's Trivacid u. a.) gefärbt. Bei der mikroskopischen Untersuchung zeigte es sich, dass die klare Flüssigkeit der ersten Probe (herausgenommen 10 Minuten nach der Injection), aus einer schwach färbbaren diffusen Masse (siehe Taf. II, Fig. 2) besteht, ohne Zweifel bei der Fixirung coagulirte Eiweissmasse. In dieser sieht man vereinzelte Diphtherie- oder Pseudodiphtherie-Bacillen, aber nichts Anderes.

Da diese klare Flüssigkeit also gar keine "Lymphkörperchen“ enthält, kann man folglich nicht annehmen, dass sie Lymphe ist, die durch Stomata des Peritonaeums in die Bauchhöhle hineingeflossen ist. Die klare Flüssigkeit muss anderswoher kommen. Mir scheint es sebr wahrscheinlich, dass sie extravasirtes Blutplasma ist. Denn man sieht auch eine sehr ähnliche Masse in der Injections-Flüssigkeit, die ausser den Bacillen nur Blutserum enthält (siehe Taf. II, Fig. 1).

In der zweiten, einige Mal erst in der dritten Probe, beginnen Leukocyten sich zu zeigen. Sie liegen da vereinzelt in der diffusen Masse. Als eine Ausnahme habe ich in einigen von meinen Probeserien schon in der ersten Probe einen oder zwei Leukocyten gesehen. Dies ist jedoch nur ein Zeichen, dass die Emigration der Leukocyten etwas früher, als gewöhnlich beginnt und ändert pichts an der Thatsache, dass im Anfang nur klare Flässigkeit und keine Lymphe in die Peritonaealhöhle hervortritt.

Allmählich werden diese Leukocyten bei jeder neuen Probe zahlreicher und zahlreicher. In den letzten Proben liegen die 
Leukocyten manchmal so dicht wie in dünnem Eiter. Ueberall sieht man auch eine gleichzeitig mit dem Auftreten der Leukocyten zunehmende Phagocytose. Die Bacillen liegen hier und da frei $z w i s c h e n$ den Leukocyten, aber zum grössten Theil sind sie in das Protoplasma der Leukocyten hineingezogen (siehe Taf. II, Fig. 3, 4, 5 und 6).

Was nun die Arten von Leukocyten betrifft, die man in den verschiedenen Proben findet, so sieht man 4 Arten von diesen. Die überwiegende Mehrzahl sind in allen Proben die polynucleären Leukocyten, aber neben diesen kommen auch, "grosse uninucleäre Leukocyten " (Taf. II, Fig. 5, M.), „Uebergangsformen" (Taf. II, Fig. 3, Ü) und kleine Lym phocyten (Taf. II, Fig. $3,4,5$ und 6, L.) vor.

Von den Lymphocyten kann man vollkommen typische Formen finden, die einen runden, homogen gefärbten Kern in der Mitte der Zelle haben, und deren Protoplasma nur einen schmalen, stark basophilen Ring vorstellt. Besonders schöne Bilder giebt Pappenheim's Methylgrün-Pyronin-Methode. In mit dieser Methode gefärbten Präparaten sieht man (Taf. II, Fig. 4, 5, 6L.) das Protoplasma der Lymphocyten sebr schön und stark roth gefärbt, während das Protoplasma der multinucleären Leukocyten schwach rosa gefärbt ist.

Neben den vollkommen typischen Lymphocyten sieht man auch Formen, wo der Kern nicht ganz rund ist und das Protoplasma breiter, als gewöhnlich. Das stark rothe, Pyronin-gefärbte Protoplasma zeigt doch immer die Lymphocyten=Natur dieser Zellen.

Die verschiedenen Arten von Leukocyten kommen in allen Proben vor, von der zweiten oder dritten, wo die Leukocyten erst hervortreten, bis zu der letzten, ungefähr 3 Stunden nach der Injection herausgenommenen. Ein bestimmtes oder regelmässig variirendes Verhältniss zwischen den Leukocyten-Arten in den verschiedenen Proben habe ich nicht feststellen können. Es scheint mir jedoch, als wären die Lymphocyten wenigstens manchmal verhältnismässig zahlreicher in den früheren, als in den späteren Proben. Immer sind die multinucleären Leukocyten sehr bedeutend zahlreicher und die anderen Arten vielmals seltener. Darum muss man auch manchmal ziemlich lange nach Lymphocyten suchen, um dieselbe zu finden. 
Phogocytose, eine Erscheinung, die man überall bei den übrigen Arten beobachtete, habe ich nur einige Male bei Lymphocyten gesehen, aber doch deutlich, sodass ich Bacillen in dem schmalen Protoplasma der Lymphocyten sitzen sah.

Ich erlaube mir hier unten zwei Versuchs Protokolle meiner Experimente mitzatheilen. Selbstverständlich ist es unnöthig, alle hier zu veröftentlichen.

Versuch von 13. März 1902. Diphtherie (Cultur 26 Stunden alt). Eine Oese mit it $\mathrm{cm}^{3}$ Serum des mit Pseudodiphtherie behandelten Kaninchens gemischt.

Original: Reinzüchtung von Diphtherie (Taf. II, Fig. 1).

6 Ubr 40 Nachm Injection in die Peritonaealhöhle eines jungen Meer* schweinchens.

1. 6 Uhr 50. Krystallklare Flüssigkeit. Mikroskopisch nur eine diffuse Masse mit vereinzelten Bacillen (Taf. II, Fig. 2).

2. 7 Uhr. Krystallklare Flüssigkeit. In der diffusen Masse sieht man vereinzelten Bacillen auch vereinzelte Leukocyten (multinucleäre und einige Lymphocyten).

3. 7 Uhr 15. Krystallklare Flüssigkeit. Die Leukocyten etwas zablreicher. Man sieht ausser multinucleären Lenkocyten auch vereinzelte uninucleäre Leukocyten, Uebergangsformen und Lymphocyten (Taf. II, Fig. 3). Beginmende Phagocytose.

4. 7 Uhr 30. Wie die vorhergebende Probe. Doch etwas zahlreicher mit Leukocyten (auch Lymphocyten zu sehen). Wie Phagocytose etwas bedeutender (Taf. II, Fig, 4).

5. 7 Ohr 45. Die Flüssigkeit beginnt träb zu werden. Die Leukocyten zablreicher.

6. 8 Uhr. Die Flüssigkeit deutlich trüb. Sehr zahlreiche multinucleăre Leukocyten. Vereinzelte uninucleäre Leukocyten und Lympho* cyten. Reichliche Phagocytose.

7. 8 Uhr 20. Trübe Flüssigkeit. Die Phagocytose noch reichlicher. Uebrigens wie die vorhergehende.

8. 8 Uhr 45. Sehr trübe Flüssigkeit. Wie die vorbergebende.

9. 9 Uhr. Wie die rorbergehende.

Versuch rom 20. März 1902. Pseudodiphtherie (Cultur 25 Stunden alt). Eine Oese der Cultur mit $\frac{x}{10} \mathrm{~cm}^{3}$ Serum des mit Pseudodiphtherie behandelten Kaninchens gemischt.

Original: Reinzüchtung von Pseudodiphtherie.

6 Uhr 25 Nachm. Injection in die Peritonaealhöhle eines jungen Meerschweinchens.

1. 6 Uhr 40. Krystallklare Flüssigkeit. Mikroskopisch nur eine diffuse Masse mit vereinzelten Bacillen.

2. 6. Uhr 50. Wie die vorbergehende Probe. Keine Leukocyten. 
3. 7 Uhr 05. Krystallklare Flüssigkeit. In der diffusen Masse neben vereinzelten Bacillen auch Leukocyten (meistens multinucleäre Leukocyten, daneben uninucleäre und Uebergangsformen, auch Lymphocyten nicht so selten). Beginnende Phagocytose.

4. 7 Uhr 20. Wie die vorhergehende. Doch etwas reichlicher Phagocytose und zablreichere Leukocyten. (Taf. II; Fig. 5.)

5. 7 Uhr 40. Die Flüssigkeit ein wenig trüb. Zahlreiche multinucleäre Leukocyten. Die äbrigen Leukocyten-Formen spärlich. Reichliche Phagocytose.

6. 8 Uhr. Die Flüssigkeit deutlich trüb. In Uebrigen wie vorhergehende.

7. 8 Uhr 15. Wie die vorhergehende.

8. 8 Uhr 30. Die Flüssigkeit sehr trüb. Sehr zahlreiche multinucleäre Lymphocyten und uninucleäre Leukocyten spärlich zu finden. Reichliche Phagocytose.

9. 8 Uhr 45. Wie die vorhergehende (Taf. II, Fig. 6).

10. 9 Uhr. Wie die vorhergehende.

11. 9 Uhr 15. Wie die vorhergehende.

12. $9 \mathrm{Uhr} 30$. Wie die vorhergehende.

Wenn man die Schnelligkeit bedenkt, mit welcher also die Lymphocyten in die Bauchhöhle bei diesen Experimenten treten (schon nach 20-40 Minuten), so ist es unmöglich, diese Zellen als "lymphocytoide" Zellen, d. h. durch Poliferation aus den fixen Zellen des Peritonaeums hervorgegangene Zellen zu betrachten. Sie müssen also ausgewanderte hämatogene Zellen sein.

Da wir weiter keinen Grund haben, einen Ausfluss der Lymphe aus den Stomata des Peritonaeums anzunehmen, sondern im Gegentheil sehen, dass die in die Peritonaealhöhle zuerst ausfliessende Flüssigkeit nicht Lymphe ist, können wir uns den Process kaum anders vorstellen, als dass die Lymphocyten durch eine active Emigration in die Bauchhöhle gelangen. $O b$ die Lymphocyten dabei aus den Blutgefässen oder aus den Lymphgefässen oder aus beiden herauswandern, lässt sich durch diese Experimente selbstverständlich nicht bestimmen. Das schadet aber Nichts. Die Hauptsache ist, dass wir kaum einen passiven Ausfluss der Lymphocyten zusammen mit auströmender Lymphe uns denken können, sondern eine active Locomobilität der Lymphocyten annehmen müssen. Und da diese Bewegung immer in einer bestimmten Richtung, nehmlich gegen die Peritonaealhöhle geschieht, kann man dieselbe nicht anders, als durch eine Chemotaxis erklären. 
Mir scheint also, dass diese Experimente sich nicht anders erklären lassen, als durch die Annahme chemotaktischer Eigenschaften und activer Emigrations-Fähigkeit sowohl der Lymphocyten, als der uninucleären und multinucleären Leukocyten.

Ioh will nur hinzufügen, dass es bei den hier vorgenommenen Experimenten scheint, als wären diese Eigenschaften der Lymphocyten weniger ausgeprägt, als die der multinucleären Leukocyten. Doch muss dies verschieden sein können, so dass für ein anderes chemotaktisches Agens die Emgration der Lymphocyten entweder grösser oder noch geringer werden könnte.

Das Resultat dieser Untersuchungen, dass die Lymphocyten eine Emigrations-Fähigkeit besitzen, steht auch nicht allein, sondern stimmt mit mehreren neueren Beobachtungen überein. So hat Hans Hirschfeld ${ }^{7}$ durch die Anwendung der von Deetjen empfohlenen Methode amöboide Beweglichkeit der Lymphocyten beobachtet, eine Eigenschaft, welche die EmigrationsFähigkeit nothwendig voraussetzt. Weiter hat A. Wolff ${ }^{17}$ einen Fall von Mastzellen und Lymphocyten in einem pleuritischen Exsudat beobachtet and er ist in diesem Fall zu dem Resultat gekommen, "dass ein chemotaktisches Agens gleichzeitig Mastzellen und Lymphocyten zur Auswanderung veranlasste."

Auch die von L. Michaelis und A. Wolff ${ }^{14}$ gemachte Beobachtung, dass Granula in dem Protoplasma der Lymphocyten vorkommen können, finde ich interessant, da dadurch der Unterschied zwischen den Lymphocyten and den übrigen Leuko* cyten auch hierin geringer wird.

\section{Erklärung der Abbildungen auf Taf. II:}

Fig. 1. Aus der Injections-Flüssigkeit $\nabla$. 13. März. Gefärbt mit Löffler's Metbylenblau. Vergrösserung $880: 1$. In einer diffusen Masse, die wahrscheinlich das coagulirte Serum ist, sieht man Mengen von Loffler's Diphtheriebacillen.

Fig. 2. Aus der ersten Probe von Versuch v. 13. März, genommen aus der Peritonaealböhle 10 Minuten nach der Injection. Gefärbt mit $L$ öf fler's Methylenblau. Vergrösserung $880: 1$. In einer diffusen Masse, die wahrscheinlich coagulirtes inflammatorisches Exsudat ist, siebt man nur vereinzelte Diphtheriebacillen (D) aber keine Leukocyten. 
Fig. 3. Aus der dritten Probe von Versuch d. 13. März, genommen aus der Peritonaealböhle 25 Minuten nach der Injection. Gefärbt mit Löffler's Methylenblau. Vergrösserung $880: 1$. In der diffusen Masse sieht man 2 multinucleäre Leukocyten (P), 1 Uebergangsform (Ü) und 2 Lymphocyten (L). Ein multinucleärer Leukocyt zeigt Phagocytose indem einige Diphtheriebacillen im Protoplasma liegen. Daneben ein vereinzelter Diphtheriebacillus (D).

Fig. 4. Aus der vierten Probe vom Versuch d. 13. März, genommen 50 Minuten nach der Injection. Färbung mit der MethylgrünPyronin-Methode. Vergrösserung $880: 1$. In der diffusen Masse sieht man einen multinucleären Leukocyt $(\mathrm{P})$, der mehrere rothe, pyroningefärbte Diphtheriebacillen in seinem Protoplasma zeigt, und einen Lymphocyt (L) mit schönem, rothem, pyroningefärbtem Protoplasma concentrisch um den blaugrünen Kern herum. Daneben einige Diphtheribacillen (D).

Fig. 5. Aus der dritten Probe vom Versuch d. 20. März, genommen aus der Peritonaealhöhle 40 Minuten nach der Injection. Fărbung mit der Methylgrün-Pyronin-Methode. Vergrösserung 880:1. In der diffusen Masse sieht man einen typischen Lymphocyten mit rothem Protoplasma und blaugrünem Kern (L), einen multinucleären Leukocyten (P) mit mebreren Pseudo-Diphtheriebacillen im Protoplasma und einen grossen uninucleären Leukocyten $(\mathbf{M})$, der auch eine reichliche Pbagocytose zeigt. Daneben vereinzelte PseudoDiphtheriebacillen (Ps).

Fig. 6. Aus der neunten Probe vom Versuch d. 20. März, genommen 2 Stunden 20 Minuten nach der Injection. Färbung mit der Methylgrün-Pyronin-Methode. Vergrösserung 880:1. In der diffusen Masse sieht man einen typischen Lymphocyten $(\mathrm{L})$ mit rothem Protoplasma und blaugrünem Kern und 9 multinucleäre Leukocyten $(\mathrm{P})$, von welchen einige Phagocytose zeigen. Daneben einige Pseudo-Diphtheriebacillen (Ps).

\section{Literatur.}

1. Baumgarten: Die Rolle der fixen Zellen in der Entzündung. Berl. klin. Wochenschr. 1900. S. 837.

2. Borst, M.: Neue Experimente zur Fremdkörperheilung. Verbandl. d. Deutschen Pathol. Gesellsch. II (München 1899). Berlin 1900.

3. von Bünger, Otto: Ueber die Einheilung von Fremdkörpern unter Einwirkung chemischer 1 . mikroparasitärer Schädlichkeiten. Beiträge z. pathol. Anat. und allgem. Pathol. Bd. 19, 1896, II.

4. Deetjen: Untersuchungen über die Blutplättchen. Dieses Archiv, Bd. 164, S. 239, 1901. 
5. Ebrlich, P. und Lazarus, A.: Die Anämie. Nothagel's Specielle Pathologie und Therapie. Bd. 8, Theil I. Heft I.

6. Hammerl, Hans: Ueber die beim Kaltblüter in Fremdkörper einwandernden Zellformen und deren weitere Schicksale. Beiträge z. patbol. Anatomie und allgem. Pathol. Bd. 19, I, 1896.

7. Hirschfeld, Hans: Sind die Lympbocyten amöboider Bewegung fähig. Berl. lilin. Wochenschr., Bd. 38, S. $1019,1901$.

8. Marchand, F.: Unterguchungen über die Einheilung von Fremdkörper. Beiträge z. patbol. Anat. u. allgem. Pathol. Bd. 4, 1889.

9. Derselbe: Referat äber die Betheiligung der Leukoeyten an der Gewebs-Neubildung, Verhandl. des 10. internat. med. Congress zu Berlin. Bd. 2, Abth. 3, S. 1, 1890.

10. Derselbe: Veber die Veränderungen der Peritonaeal-Epithelien (Deckzellen) bei der Einheilung kleiner Fremdkörper. Sitzungsbericht der Geselischaft zur Bef. der Naturwissenschaft zu Marburg. 1897. No. 3.

11. Derselbe: Ueber die Bedeutung der grosşkernigen Wanderzellen bei der durch Einfübrung kleiner Fremdkörper in die Bauchhöhle erzeugten Entzündung. Daselbst No. 6 .

12. Derselbe: Ueber die bei Entzündung in der Peritonaealböhle anftretende Zellformen. Verhandl. der deutschen pathol. Gesellsch. (Düsseldorf) I. Berlin 1899.

13. Derselbe: Der Process der Wundbeilung. Deutsche Chirurgie. 1901. 14. Michaelis, L. und Wolff, A.: Ueber Granula in Lymphocyten. Dieses. Archiv, Bd. 167, Heft 1, S. 151. 1902.

15. Pappenheim, A.: Ueber das Vorkommen einkerniger Zellen im gonorhoischen Urethral-Secret. Dieses Archiv, Bd. 164, S. 72, 1901.

16. Derselbe: Wie verhalten sich die Unna'schen Plasmazellen zu Lymphocyten. Dieses Archir, Bd. 165, S. 365, 1901 und Bd. 166, 1901.

17. Wolff, A.: Ueber Mastzellen in Exsudaten. Ein Beitrag zur Frage der aktiven Lymphocytose. Münchener medicinische Wochenschrift. No.6. 1902 . 